\title{
Geometric Classification of Paraclinoid Aneurysms for Microcatheter Superselection in Coil Embolization
}

\author{
Yeongu CHUNG ${ }^{1}$, Jiwook RYU², Eui Jong $\mathrm{KIM}^{3}$, Sung Ho LEE ${ }^{4}$, Seok Keun $\mathrm{CHOI}^{2}$ \\ ${ }^{1}$ Sungkyunkwan University School of Medicine, Kangbuk Samsung Hospital, Department of Neurosurgery, Seoul, Korea \\ ${ }^{2}$ Kyung Hee University, College of Medicine, Kyung Hee University Hospital, Department of Neurosurgery, Seoul, Korea \\ ${ }^{3}$ Kyung Hee University, College of Medicine, Kyung Hee University Hospital, Department of Radiology, Seoul, Korea \\ ${ }^{4}$ Seoul National University College of Medicine, Seoul National University Hospital, Department of Neurosurgery, Seoul, Korea \\ Corresponding author: Seok Keun CHOI nscsk@hanmail.net
}

\section{ABSTRACT}

AIM: To suggest a geometric classification of paraclinoid aneurysms for microcatheter superselection.

MATERIAL and METHODS: Clinical data from 76 patients (80 paraclinoid aneurysms) who underwent endovascular treatments were retrospectively reviewed. Paraclinoid aneurysms were classified according to the six directions where the aneurysm neck lies and simplified into three groups as follows: superior, medial, and lateral groups. The medial group was further divided into proximal, mid, and distal subgroups according to the location of the aneurysm neck on lateral angiography. Furthermore, we assessed the superselection success rate with the first-selected pre-shaped microcatheter per group.

RESULTS: According to the aneurysm direction, the medial group showed relatively lower superselection success rates (66.1\%) than the superior (81.8\%) and lateral groups (85.7\%). The S-shaped microcatheter was the most frequently used in the superior $(69.2 \%)$ and lateral groups (62.5\%). Acute-angled $\mathrm{J}$ - and C-shaped microcatheters (88.5\%) were preferred for proximal aneurysms; and obtuse-angled $45^{\circ}$ - and $90^{\circ}$-angled microcatheters $(75 \%)$, for distal aneurysms. The mid-portion group showed the lowest success rate $(45.8 \%)$ and more difficulties in pre-shaped microcatheter superselection.

CONCLUSION: Medially directed mid-portion aneurysms were difficult to access using pre-shaped microcatheters; thus, tailored steam-shaping techniques may be considered. Superiorly and laterally directed aneurysms could be accessed using pre-S-shaped microcatheters. Acute-angled microcatheters may be considered for proximal aneurysms; and obtuse-angled microcatheters, for distal aneurysms.

KEYWORDS: Endovascular procedures, Internal carotid artery, Intracranial aneurysm

ABBREVIATIONS: AP: Anteroposterior, DSA: Digital subtraction angiography, ICA: Internal carotid artery

\section{INTRODUCTION}

$\mathrm{P}$ araclinoid aneurysms can generally be defined as intracranial aneurysms arising from the internal carotid artery (ICA) between the portion distal to the roof of the cavernous sinus and the origin of the posterior communicating artery $(7,12,14)$. Endovascular coil embolization has emerged as an alternative treatment modality for surgical clipping of intracranial aneurysms. Recently, advanced techniques and developed interventional devices have enabled coil embolization of geometrically difficult aneurysms, including paraclinoid aneurysms $(3,10,11)$. Nevertheless, managing paraclinoid aneurysm is still challenging, and these lesions remain difficult to treat either surgically or endovascularly. Owing to the vascular tortuosity and complex anatomical characteristics of the carotid siphon, paraclinoid aneurysms have been one of the most technically inaccessible lesions.
Yeongu CHUNG (1) : 0000-0001-9139-0988

Jiwook RYU (1): 0000-0001-5091-6760

Eui Jong KIM (1): 0000-0003-2183-8657
Sung Ho LEE (10): 0000-0002-0202-8969

Seok Keun CHOI (D) : 0000-0003-0702-8463 
To date, various methods that facilitate superselection of paraclinoid aneurysms have been reported $(4,10)$. Furthermore, several reports have classified paraclinoid aneurysms according to aneurysm type or direction $(2,12,14)$. However, most reports used anatomical classifications for the purpose of surgical clipping or angiographically simple classifications according to location. Therefore, we suggest a more detailed geometric classification based on digital subtraction angiography (DSA) findings for Microcatheter superselection with less difficulty and complexity. Herein, we assessed a classification of paraclinoid aneurysms and analyzed the success rate for aneurysm selection using recently produced pre-shaped microcatheters according to their type.

\section{MATERIAL and METHODS}

\section{Population}

Between April 2009 and October 2016, 342 intracranial aneurysms were treated with coil embolization at our institute. The inclusion criteria included paraclinoid ICA aneurysms treated with coil embolization or stent-assisted coil embolization within the study period. Paraclinoid ICA aneurysms were defined as intracranial ICA aneurysms between the clinoid segment and the communicating segment of the ICA. The exclusion criteria included cavernous ICA aneurysms proximal to the carotid siphon or giant aneurysms. A total of 76 patients (80 paraclinoid ICA aneurysms) were included in this study. Medical and radiographic records, and data from an endovascular procedure database were retrospectively reviewed. The baseline characteristics of the patients with paraclinoid ICA aneurysms are summarized in Table I. The institutional review board approved this retrospective study and waived the need for informed patient consent.

\section{Endovascular Procedure}

All the patients underwent pre-procedural DSA, and rotational three-dimensional reconstruction images were obtained using a biplanar angiographic unit (Allura Xper: Philips Medical Systems, Best, Netherlands) for the evaluation of aneurysm characteristics (i.e., diameter, neck width, directions, and locations). All the patients were given antiplatelet agents before coil embolization in accordance with the protocol of our institution. All the procedures were performed under general anesthesia. A 6-Fr guide catheter (Envoy, Codman Neurovascular, Raynham, MA, USA) was placed in the proximal ICA near the carotid canal in all the cases. Thereafter, properly selected pre-shaped microcatheters (Excelsior SL-10, Stryker Neurovascular, Fremont, CA, USA; Prowler Select Plus: Codman Neurovascular, Raynham, MA, USA) were introduced over a micro-guidewire and navigated into the aneurysm sac under fluoroscopy. Detachable coil embolization with or without stent assistance was performed using various types of coil. We defined first-trial success as the presence of superselection using the first-selected preshaped microcatheter into the aneurysm sac. We excluded the cases of changing to other pre-shaped microcatheters, tailored steam shaping, or balloon assistance from among the cases of first-trial success. However, we included the cases that needed double microcatheters only for microcatheter stabilization and had a successful navigation. Although considering that microcatheter stabilization is also important for coil embolization, we focused only on easy navigation and superselection of microcatheters into the aneurysm sac.

\section{Assessment of Aneurysm Direction}

The paraclinoid aneurysms were classified according to the direction in which the aneurysm neck lies. We evaluated the direction of the virtual line that crosses between the centers of the aneurysm sac and aneurysm neck in the anteroposterior (AP) view of the DSA conducted by a single neurosurgeon (Y.C.). We categorized the aneurysms according to the angle formed between the horizontal and virtual lines in six directions as follows: superior, superomedial, medial, inferomedial, inferior, and lateral (Figure 1). These six directions were simplified and classified into the following three groups for the assessment of the used pre-shaped microcatheter per group: 1) superior group, including the superior and superomedial directions; 2) medial group, including the medial, inferomedial, and inferior directions; 3) lateral group, including the lateral direction.

\section{Assessment of Aneurysm Location}

The length and curve of the carotid siphon were considered

Table I: Characteristics of the Patients with Paraclinoid Internal Carotid Artery Aneurysms

\begin{tabular}{lc}
\hline Characteristics & Value \\
\hline No. of patients and aneurysms & $\begin{array}{c}76 \text { patients, } 80 \\
\text { aneurysms }\end{array}$ \\
\hline Sex, $\mathrm{n}(\%)$ & $16(20.0)$ \\
\hline Male & $64(80.0)$ \\
\hline Female & $58.8(30-83)^{\star}$ \\
\hline Mean age, years (range) & $25(31.2)$ \\
\hline Endovascular treatment, $\mathrm{n}(\%)$ & $55(68.8)$ \\
\hline Coil embolization without stent & \\
\hline Stent-assisted coil embolization & $33: 47^{*}$ \\
\hline Aneurysmal characteristics & \\
\hline Location $(\mathrm{n}$, right: left) & $55(68.7)$ \\
\hline Size, $\mathrm{n}(\%)$ & $24(30.0)$ \\
\hline Tiny $(<5 \mathrm{~mm})$ & $1(1.3)$ \\
\hline Small $(5-10 \mathrm{~mm})$ & \\
\hline Large $(>10 \mathrm{~mm})$ & $60(75.0)$ \\
\hline Neck width, $\mathrm{n}(\%)$ & $20(25.0)$ \\
\hline Narrow $(<4 \mathrm{~mm})$ & \\
\hline Wide $(>4 \mathrm{~mm})$ & \\
\hline
\end{tabular}

"Other values 


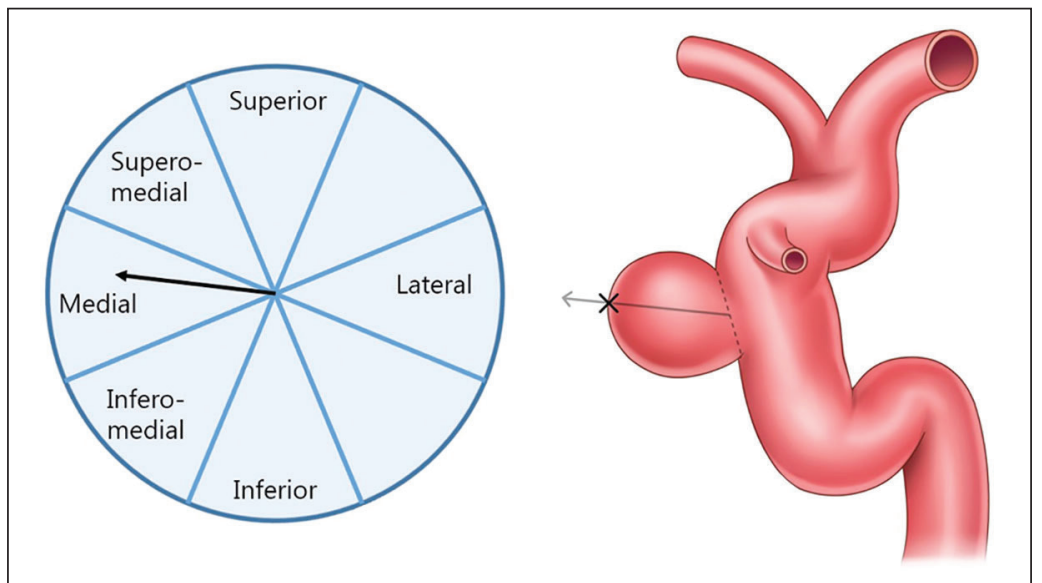

Figure 1: Assessment method for paraclinoid aneurysms according to the following six directions (angle formed between the horizontal and virtual lines from the center point of the aneurysm neck to the dome): superior, superomedial, medial, inferomedial, inferior, and lateral.

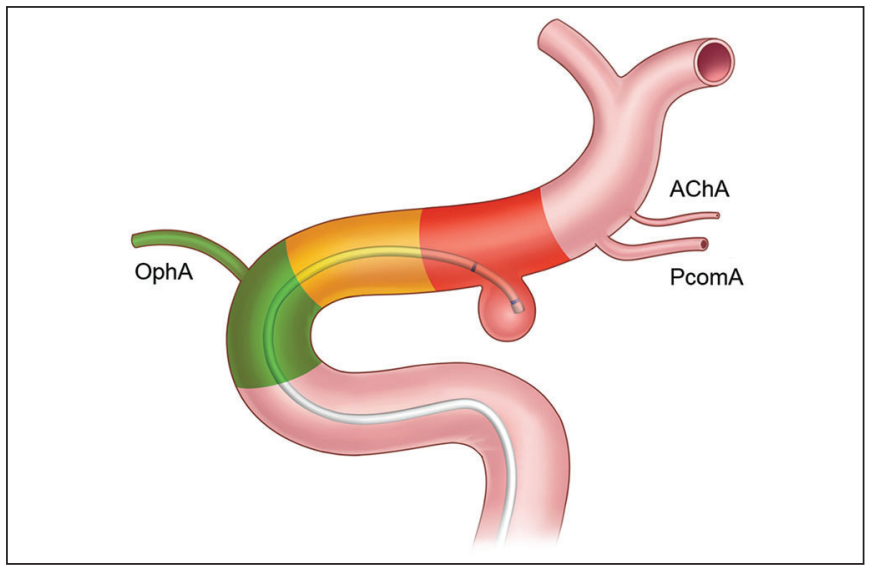

Figure 2: Schematic illustration of the assessment method for paraclinoid aneurysms according to their location. We classified the paraclinoid aneurysms into three groups (proximal, mid, and distal portions) according to the relative anatomical location of the aneurysm neck origin. AChA: Anterior choroidal artery, OphA: ophthalmic artery, PcomA: posterior communicating artery.

critical factors for selecting the appropriate pre-shaped microcatheter. Thus, we further divided the paraclinoid aneurysms into three groups (proximal, mid, and distal portions) according to their relative anatomical location as shownin the lateral view of the DSA. We separated them via imaginary trisection from the clinoid segment of the ICA, which started from the distal portion of the anterior genu to the origin of the posterior communicating artery. Thereafter, we classified them according to the origin of the central point of the aneurysm neck (Figure 2).

\section{Aneurysm Classification}

The classifications according to aneurysm direction and location are summarized in Table II. Among 80 paraclinoid aneurysms, $11(13.8 \%)$ were included in the superior group; $62(77.5 \%)$, in the medial group; and 7 (8.7\%), in the lateral group (Figure 1). The superior group included ophthalmic artery and dorsal wall paraclinoid aneurysms. The medial group, which had the largest number of aneurysms, included most of the superior hypophyseal artery aneurysms. Among
Table II: Paraclinoid Aneurysms Grouped by Direction and Location

\begin{tabular}{cccc}
\hline & Proximal & Mid & Distal \\
\hline Superior group & & & \\
\hline Superior & 2 & 1 & 2 \\
\hline Superomedial & 2 & 2 & 2 \\
\hline Medial group & & & \\
\hline Medial & 7 & 8 & 3 \\
\hline Inferomedial & 22 & 12 & 2 \\
\hline Inferior & 3 & 4 & 1 \\
\hline Lateral group & & & \\
\hline Lateral & 7 & N/A & N/A \\
\hline
\end{tabular}

N/A: not available.

the aneurysms, $43(53.7 \%)$ were in the proximal portion; $27(33.8 \%)$, in the midportion; and $10(12.5 \%)$, in the distal portion.

\section{Pre-shaped Microcatheters}

We used 2 brands of braided microcatheters (Excelsior SL-10 and Prowler Select Plus). Aside from the straight microcatheters, the available pre-shaped microcatheters included the $45^{\circ}$ - and $90^{\circ}$-angled, and $\mathrm{J}$-, C-, and S-shaped microcatheters. In cases of failure to navigate using the pre-shaped microcatheters, tailored steam shaping of the microcatheters was performed according to each arterial course. Steam-shaping techniques such as the pigtail technique provide three-dimensional shapes to follow the tortuosity of the ICA and carotid siphon (10). For the evaluation of the success rate of superselection using pre-shaped microcatheters, we classified the pre-shaped microcatheters into the following three groups: 1) obtuse-angled microcatheters, including $45^{\circ}$ - and $90^{\circ}$-angled $\left(\leq 90^{\circ}\right)$ microcatheters; 2) acute-angled microcatheters, including the $\mathrm{J}$ - and $\mathrm{C}$-shaped $\left(>90^{\circ}\right)$ microcatheters; and 3) S-shaped microcatheters. 


\section{Aneurysm Size}

We evaluated the correlation between the success rate of aneurysm superselection and size. We measured both the maximal diameter of the aneurysm sac and neck width. Thereafter, the aneurysm size between the first-trial success group as mentioned above and the other groups was compared.

\section{Statistical Analyses}

When bilateral aneurysms were present in the same patients, they were assessed independently. Statistical analysis was performed using SPSS 17.0 (Chicago, IL, USA). The Fisher exact test was used to compare the first-trial success rate with the recommended pre-shaped microcatheters among the groups.

\section{RESULTS}

\section{Patients and Aneurysms}

The general characteristics of the enrolled patients are summarized in Table I. Bilateral superior hypophyseal artery aneurysms were found in 4 patients $(5 \%)$ who were treated with endovascular treatment for both lesions. The mean \pm SD age of the included patients was $58.8 \pm 10.7$ years. Of the patients, $16(20 \%)$ were men and $64(80 \%)$ were women. Twenty-five patients (31.2\%) underwent coil embolization without a stent, and 55 (68.8\%) underwent stent-assisted coil embolization. The aneurysms were located on the right ICA in 33 patients and left ICA in 47 patients. The mean \pm SD size and neck width of 80 aneurysms were $4.54 \pm 1.77$ and $3.33 \pm$ $1.25 \mathrm{~mm}$, respectively.

\section{Alternative Techniques in Case of Failure of Superselection with the First-selected Microcatheters}

For cases with difficult microselection of aneurysms or lack of microcatheter stabilization in the aneurysm sac, additional techniques were performed in 34 patients (42.5\%) as follows: changing the microcatheter in 14 patients (17.5\%), tailored steam shaping in 8 patients $(10.0 \%)$, balloon remodeling in 2 patients $(2.5 \%)$, and double microcatheter technique in 10 patients (12.5\%).

\section{Success Rate of Superselection According to Aneurysm Direction}

We evaluated the success rate of superselection with the first-selected pre-shaped microcatheter per group according to aneurysm direction (Figure $3 \mathrm{~A}-\mathrm{C}$ ). In the superior group, 9 (81.8\%) of the 11 patients achieved successful superselection; among the finally selected microcatheters, 2 were $45^{\circ}$-angled microcatheters; 2, J-shaped microcatheters; and 9, S-shaped microcatheters. In the medial group, 41 (66.1\%) of the 62 patients achieved successful superselection. Among the patients with failure of superselection, 12 underwent changing of the microcatheter with another pre-shaped microcatheter; 8 , steam shaping; and 1, balloon-assisted technique. Among the finally selected microcatheters, 7 were $45^{\circ}$-angled microcatheters; 7, 90-angled microcatheters; 25, J-shaped microcatheters; and 8, C-shaped microcatheters. In the lateral group, 6 (85.7\%) of the 7 patients achieved successful superselection. Among the finally selected microcatheters, one was a $45^{\circ}$-angled microcatheter; 2 , J-shaped microcatheters; and 5, S-shaped microcatheters. The S-shaped microcatheter was preferred, especially in the superior (69.2\%) and lateral groups (62.5\%).

\section{Success Rate of Superselection According to Aneurysm Location}

Although the medial group showed the highest incidence rate $(77.5 \%)$, they had a relatively lower success rate $(66.1 \%)$ of superselection than the superior (81.8\%) and lateral groups (85.7\%). Thus, we performed an additional analysis of success rate according to the aneurysm location in the medial group (Figure $4 \mathrm{~A}-\mathrm{C}$ ). Of the 32 proximal aneurysms, $24(75.0 \%)$ achieved successful superselection with the firstselected microcatheters; among the microcatheters, 2 were $45^{\circ}$-angled microcatheters, 1 was a 90 -angled microcatheter, 15 were J-shaped microcatheters, and 8 were C-shaped microcatheters. The acute-angled (J and C, 88.5\%) pre-shaped microcatheters were preferred for the proximal aneurysms. Of the 24 mid-portion aneurysms, 11 (45.8\%) achieved successful superselection; among the microcatheters, 2 were $45^{\circ}$-angled microcatheters, 3 were $90^{\circ}$-angled microcatheters, 8 were J-shaped microcatheters, and 1 wasa C-shaped microcatheter. Of the distal aneurysms, all 8 (100\%) achieved superselection success (three $45^{\circ}$-angled, three $90^{\circ}$-angled, and two J-shaped microcatheters). The obtuse-angled $\left(45^{\circ}\right.$ and $90^{\circ}, 75 \%$ ) pre-shaped microcatheters were preferred for the distal-portion aneurysms. The mid-portion group showed the lowest success rate among the groups. Furthermore, 6 $(75.0 \%)$ of the 8 tailored steam-shaped cases were included

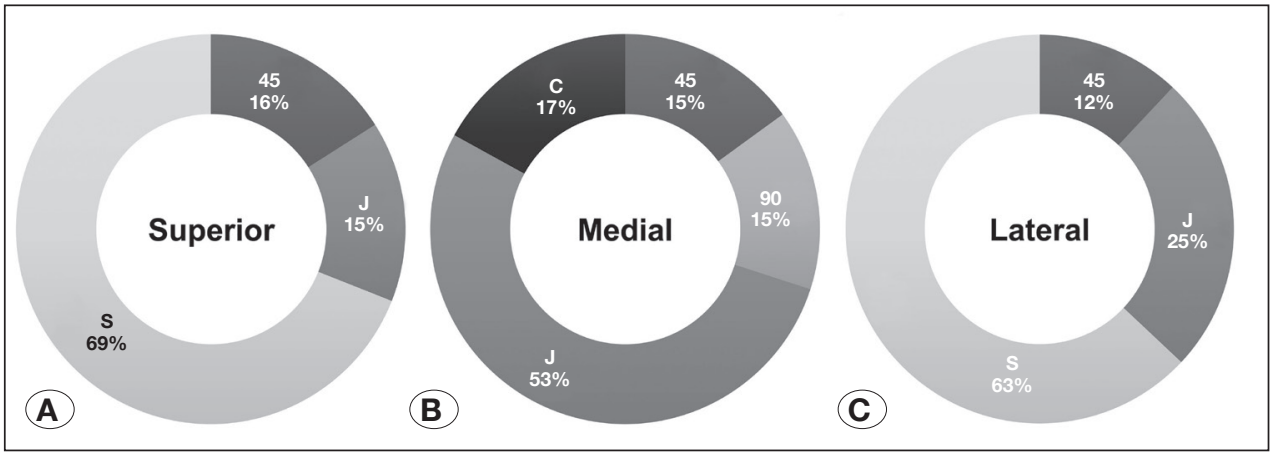

Figure 3: Diagram showing the type of first-selected pre-shaped microcatheter with successful aneurysm superselection in each aneurysm direction group: A) superior group, B) medial group, and C) lateral group.The S-shaped microcatheter was preferred especially in the superior (69.2\%), and lateral groups (62.5\%). 
in the mid-portion subgroup of the medial group. More difficulties in microcatheter superselection were encountered in the medial group (according to aneurysm direction) and mid-portion group (according to aneurysm location).

\section{Comparison of the First-trial Success Rate of Superselection}

On the basis of the results and analyses, we suggest a strategy for pre-shaped microcatheter superselection during endovascular treatment of paraclinoid aneurysms (Figure 5). We found that the first-trial success rate of microselection was higher when these recommendations were followed with the use of the pre-shaped microcatheters (Figure 6). In the superior group, the first-trial success rate was $87.5 \%(7 / 8)$ with the recommended microcatheters and $80 \%(4 / 5)$ with the other microcatheters. The success rates in the lateral group were $100 \%(5 / 5)$ and $75 \%(3 / 4)$, respectively. The proximal subgroup of the medial group showed success rates of $85.2 \%(23 / 27)$ and $42.9 \%(3 / 7)$, respectively, which were statistically significant $(p=0.037)$. Finally, the distal subgroup of the medial group showed a success rate of $100 \%$ for both the recommended and other microcatheters. All the subgroup analyses showed high success rates.

\section{Aneurysm Size}

We divided the patients into 2 groups, a first-trial success group and a superselection failure group. In the first-trial success group, the mean sizes of the aneurysms were $4.47 \mathrm{~mm}$ (diameter) and $3.42 \mathrm{~mm}$ (neck width). In the superselection failure group, the mean sizeswere $4.71 \mathrm{~mm}$ (diameter) and $3.11 \mathrm{~mm}$ (neck width). The larger and narrower neck aneurysms showed more difficulties in superselection without significance. Among the patients with an aneurysm neck width of $<4 \mathrm{~mm}, 38$ (63.3\%) underwent stent-assisted coil embolization. Conversely, among the patients with a neck width of $>4 \mathrm{~mm}, 18(90.0 \%)$ underwent stent-assisted coil embolization.

\section{DISCUSSION}

\section{Considerations for Coil Embolization of Paraclinoid Aneurysms}

Various considerations for coil embolization of cerebral aneurysms are required, such as three-dimensional vascular anatomy around the aneurysm and the characteristics of devices. Paraclinoid aneurysms are one of the most technically inaccessible lesions via the endovascular approach owing to
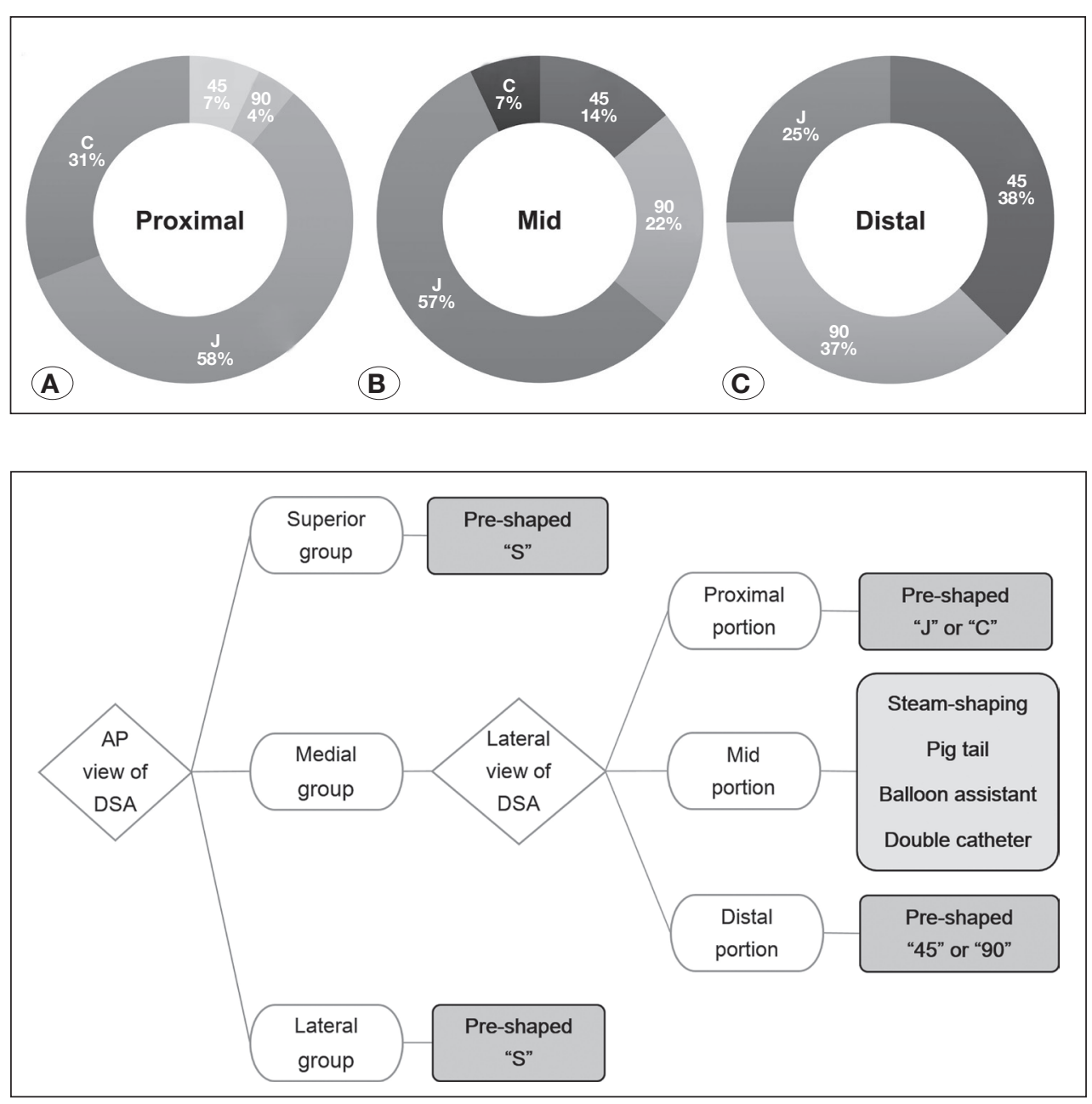

Figure 4: Diagram showing the type of first-selected pre-shaped microcatheter with successful aneurysm superselection in each group according to aneurysm location. A) Proximal portion: The acute-angled (J plus C, 88.5\%) pre-shaped microcatheters were preferred in this group. B) Midportion. C) Distal portion: The obtuse-angled $\left(45^{\circ}\right.$ plus $\left.90^{\circ}, 75 \%\right)$ pre-shaped microcatheters were preferred in this group.

Figure 5: Suggested strategy for the selection of pre-shaped microcatheters. AP: anteroposterior, DSA: digital subtraction angiography 


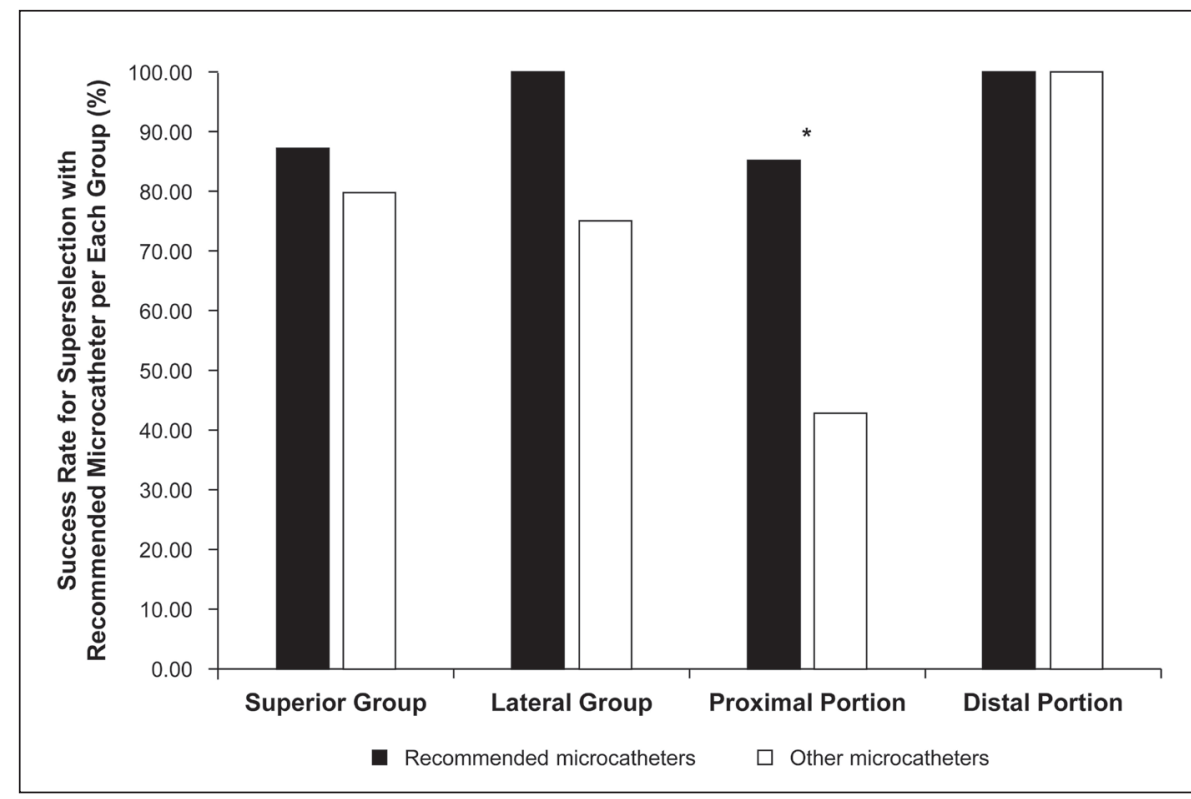

Figure 6: Comparison of first-trial success rate for superselection among the recommended pre-shaped microcatheters per group. The proximal subgroup of the medial group showed success rates of $85.2 \%(23 / 27)$ and $42.9 \%(3 / 7)$ for the recommended and other microcatheters, which were statistically significant $\left({ }^{*} \mathrm{p}=0.037\right)$. the difficult arterial anatomy of the carotid siphon. Cho et al. reported that the 2 major technical issues in the treatment of these lesions include microcatheter aneurysm selection and the kickback during coil insertion (4). The position of the tip of the microcatheters must be kept in the center of the aneurysm (13). In other words, both superselection and stabilization of microcatheters are major concerns for treatment. Superselection of microcatheters according to the appropriate position of aneurysms is important as an initial procedure. Therefore, some authors have reported shaping, navigating, or looping techniques of microcatheters in coil embolization of paraclinoid aneurysms $(3,4,10)$. For appropriate selection of microcatheters, microcatheter shifting or micro-guide wire steering techniques are also important (10). Even with these techniques, appropriate microcatheter superselection takes precedence. The appropriate shape of microcatheters is important not only for selecting aneurysms but also for achieving high stability. Furthermore, the currently used preshaped microcatheters can solve the problems from tailored steam shaping for beginner endovascular neurosurgeons. Our suggestion according to the simple geometric classification of paraclinoid aneurysms may offer easier methods for selecting appropriate pre-shaped microcatheters and may reduce unnecessary time-consuming procedures. Adjunctive techniques during the procedure may increase the frequency of thromboembolic complications (15).

\section{Classification of Paraclinoid Aneurysms}

Since Kothandaram et al. first proposed the classification of paraclinoid aneurysms based on intraoperative findings (8), several classifications based on angiographic findings have also been introduced $(1,2,5,6,9)$. Most reported classification systems were created for neurosurgical purposes before the era of endovascular treatment but are impractical to apply in endovascular treatment. Therefore, we classified these lesions on the basis of the more detailed angiographic geometry for microcatheter superselection. Some authors have reported classification-based microcatheter-shaping methods and outcomes (14). They classified paraclinoid aneurysms into 4 subtypes and recommended the appropriate microcatheter shapes for them. Our classification system divides paraclinoid aneurysms into two categories according to the AP and lateral views of DSA, with each category divided further into three subtypes. We suggest a more detailed classification because the geometric direction and location of aneurysms are important considerations for microcatheter superselection. First, we classified these lesions into six directions according to the direction in which the aneurysm neck lies in the AP view and simplified them into 3 groups as follows: superior (superior and superomedial directions), medial (medial, inferomedial, and inferior directions), and lateral groups (lateral direction). Next, we classified them by location in the lateral view into three groups (proximal, mid, and distal portions) for the assessment of the success rate of aneurysm superselection with the selected pre-shaped microcatheter per group. Our classification system is simple and can easily assess aneurysms using DSA. The detailed two-dimensional geometric subgroups could be helpful for the selection of an appropriate microcatheter during endovascular treatment.

\section{Suggested Strategy for Selection of Pre-shaped Microcatheters}

In our study, the superior and lateral groups showed higher success rates using the first-selected pre-shaped microcatheters than the medial group $(81.8 \%$ and $85.7 \%$ vs $66.1 \%)$. Most of these groups showed the highest success rate using the pre-S-shaped microcatheter (Figure 3). A microcatheter with a certain shape was designed along the curvature of the carotid siphon especially to rest on the inferior wall of the ICA segment (10). Although the medial group showed the highest prevalence $(77.5 \%)$, they showed the lowest success rate of microcatheter superselection 
(66.1\%). Therefore, we performed an additional dimensional analysis for the medial group. The proximal and distal groups showed relatively higher success rates than the midportion group (75\% and $100 \%$, respectively, vs $45.8 \%)$. The proximal group showed a success rate of $88.5 \%$ with the first-selected pre-J- and pre-C-shaped microcatheters, which are more acute-angled microcatheters. The distal group showed a success rate of $75 \%$ with the first-selected $45^{\circ}$ and $90^{\circ}$-angled microcatheters. The medial group in the AP view and mid-portion group in the lateral view of DSA showed the greatest difficulty in microcatheter superselection. When selecting microcatheters for these groups, various shaping and navigating techniques must be considered. With this analysis, we suggest our strategy for selection of pre-shaped microcatheters during endovascular treatment of paraclinoid aneurysms (Figure 5). We also retrospectively analyzed the first-trial success rate and confirmed the higher success rate (Figure 6). This strategy might help select the appropriate pre-shaped microcatheter for beginner endovascular neurosurgeons.

\section{CONCLUSION}

Our analysis according to the geometric classification of paraclinoid aneurysms revealed that medially directed midportion aneurysms were more difficult to access using preshaped microcatheters. Tailored steam-shaping techniques may be considered for these paraclinoid aneurysms. Superiorly and laterally directed aneurysms could be accessed using pre-S-shaped microcatheters. Acute-angled microcatheters may be considered for proximal aneurysms; and obtuseangled microcatheters, for distal aneurysms.

\section{ACKNOWLEDGEMENTS}

We would like to thank Editage (www.editage.co.kr) for English language editing.

\section{REFERENCES}

1. al-Rodhan NR, Piepgras DG, Sundt TM Jr: Transitional cavernous aneurysms of the internal carotid artery. Neurosurgery 33:993-998, 1993

2. Barami K, Hernandez VS, Diaz FG, Guthikonda M: Paraclinoid carotid aneurysms: Surgical management, complications, and outcome based on a new classification scheme. Skull Base 13:31-41, 2003

3. Cho YD, Kang HS, Kim JE, Son YJ, Lee JY, Lee SJ, Seo JH, Han MH: Microcatheter looping technique for coil embolization of complex configuration middle cerebral artery aneurysms. Neurosurgery 71:1185-1191, 2012
4. Cho YD, Rhim JK, Park JJ, Jeon JS, Yoo RE, Kang HS, Kim JE, Cho WS, Han MH: Microcatheter looping to facilitate aneurysm selection in coil embolization of paraclinoid aneurysms. Korean J Radiol 16:899-905, 2015

5. Day AL: Aneurysms of the ophthalmic segment. A clinical and anatomical analysis. J Neurosurg 72:677-691, 1990

6. De Jesus O, Sekhar LN, Riedel CJ: Clinoid and paraclinoid aneurysms: Surgical anatomy, operative techniques, and outcome. Surg Neurol 51:477-488, 1999

7. Hoh BL, Carter BS, Budzik RF, Putman CM, Ogilvy CS: Results after surgical and endovascular treatment of paraclinoid aneurysms by a combined neurovascular team. Neurosurgery 48:78-90, 2001

8. Kothandaram P, Dawson BH, Kruyt RC: Carotid-ophthalmic aneurysms. A study of 19 patients. J Neurosurg 34:544-548, 1971

9. Kumon Y, Sakaki S, Kohno K, Ohta S, Ohue S, Oka Y: Asymptomatic, unruptured carotid-ophthalmic artery aneurysms: Angiographical differentiation of each type, operative results, and indications. Surg Neurol 48:465-472, 1997

10. Kwon BJ, Im SH, Park JC, Cho YD, Kang HS, Kim JE, Han $\mathrm{MH}$ : Shaping and navigating methods of microcatheters for endovascular treatment of paraclinoid aneurysms. Neurosurgery 67:34-40, 2010

11. Malek AM, Halbach VV, Phatouros CC, Lempert TE, Meyers PM, Dowd CF, Higashida RT: Balloon-assist technique for endovascular coil embolization of geometrically difficult intracranial aneurysms. Neurosurgery 46:1397-1407, 2000

12. Park HK, Horowitz M, Jungreis C, Kassam A, Koebbe C, Genevro J, Dutton K, Purdy P: Endovascular treatment of paraclinoid aneurysms: Experience with 73 patients. Neurosurgery 53:14-24, 2003

13. Suzuki S, Kurata A, Ohmomo T, Sagiuchi T, Niki J, Yamada M, Oka H, Fujii K, Kan S: Endovascular surgery for very small ruptured intracranial aneurysms. Technical note. J Neurosurg 105:777-780, 2006

14. Wang $Y$, Li $Y$, Jiang $C$, Wu Z, Jiang F, Meng $H$, Siddiqui $A H$, Yang $X$ : Could the types of paraclinoid aneurysm be used as a criterion in choosing endovascular treatment? Neuroradiologists' view. Acta Neurochir (Wien) 155:2019-2027, 2013

15. Yonaha H, Hyodo A, Inaji T, Ito K, Kushi S, Tsuchida K, Saito A, Sugimoto K, Yoshii Y: Thromboembolic events associated with coil protrusion into parent arteries after GDC treatment. Interv Neuroradiol 12:105-111, 2006 